\title{
Limitations of the Barcelona clinic liver cancer staging treatment strategy in hepatocellular carcinoma patients with performance status 1
}

\author{
Jung Hwan Yu, Jin-Woo Lee \\ Department of Internal Medicine, Inha University Hospital and School of Medicine, Incheon, South Korea \\ Correspondence to: Jin-Woo Lee, MD, PhD. Professor of Medicine, Division Chief of Gastroenterology and Hepatology, Inha University Hospital, 27 \\ Inhang-Ro, Jung-Gu, Incheon 22332, South Korea. Email: jin@inha.ac.kr. \\ Provenance and Peer Review: This article was commissioned by the editorial office, Annals of Translational Medicine. The article did not undergo \\ external peer review. \\ Comment on: Zhao S, Dou W, Fan Q, et al. Identifying optimal candidates of transarterial chemoembolization (TACE) vs. sorafenib in patients with \\ unresectable hepatocellular carcinoma. Ann Transl Med 2020;8:587.
}

Submitted May 28, 2020. Accepted for publication Jun 23, 2020.

doi: $10.21037 /$ atm-20-4316

View this article at: http://dx.doi.org/10.21037/atm-20-4316

Hepatocellular carcinoma (HCC) is the sixth most common cancer worldwide and is the leading cause of death in patients with cirrhosis (1). Patients at an early stage of HCC can be cured by treatments such as surgery, liver transplantation, and radiofrequency ablation (RFA). However, in advanced HCC, a standard treatment has not yet been established. Also, the prognosis is generally poor despite various available treatments including transcatheter arterial chemoembolization (TACE), radiation therapy, and systemic chemotherapy (2). Therefore, for patients with advanced HCC, it is necessary to establish a treatment plan considering the patient's age, tumor stage and performance status (PS).

PS reflecting the patient's general well-being and activities of daily life is a key factor in determining the prognosis and treatment plan for cancer patients. Nevertheless, the importance of PS in clinical practice tends to be overlooked. According to a study in Japan that investigated the relationship between PS and HCC prognosis in 1,003 patients, the overall survival (OS) rates at 1,3 , and 5 years after each initial treatment were significantly higher in patients with PS 0 than in PS 1 or 2 (3). In another study of 2,381 HCC patients, the long-term survival tended to be worse in patients with progressively poor PS (all $\mathrm{P}<0.05$ ), and PS was found to be an independent prognostic predictor of HCC patient survival (4). Therefore, measurement of individual PS is very crucial to predict treatment outcome for HCC patients.

Barcelona clinic liver cancer (BCLC) staging is currently the most widely used treatment algorithm for HCC, and it requires patients with PS 1 or higher to use Sorafenib or choose supportive care. However, recent studies show that HCC patients with PS 1 or higher also have better prognosis when treated more actively (5-7). In a study of 2,620 HCC patients with PS 1-2 by Hsu et al., patients who received active treatments including surgery, reported a statistically significant survival advantage compared to those who followed the BLCL guidelines $(\mathrm{P}<0.001)(7)$. This study (Zhao et al.) also reported a better prognosis in patients treated with TACE than those treated with sorafenib according to the BCLC guideline in the absence of vascular invasion or metastasis in BCLC stage C patients with PS 1 (8). Thus, in patients with BCLC stage $\mathrm{C}$, choosing an optimal treatment strategy based on the objective measurement of PS could get a better clinical outcome rather than following the BCLC guidelines.

As many systemic chemotherapeutic agents for HCC have been recently developed, there is a trend favoring active use of these drugs. However, the newly developed drugs did not show superior treatment results compared to the widely used sorafenib and is not effective for all advanced HCC patients (9). Of course, since various chemotherapies combined with immune checkpoint 
inhibitors are under study, better systemic chemotherapy may be available in the future (10). Sometimes in patients with high risk of surgical resection, loco-regional treatment such as RFA, TACE, and sometimes other treatments combined with radiation therapy may improve the prognosis. Therefore, selecting the right treatment for each patient is of utmost importance.

As reported by Zhao et al., if patients in BCLC stage $C$ with PS 1 do not have vascular invasion or extrahepatic spread, more aggressive locoregional therapy could be attempted before choosing a systemic chemotherapy such as sorafenib (8). Furthermore, adjuvant radiotherapy or surgical resection will be an effective treatment option for carefully selected patients. In this regard, we have some questions about this paper (Zhao et al.). First, the target patients were defined as unresectable, but it is unclear whether these patients were unsuitable for surgery. This is because many patients had a single tumor lesion $(49.9 \%$, 161/323) and good liver function of CTP class A (92\%, 297/323). As one of major limitations, it is possible that this study included cases where TACE was performed for a variety of reasons even in patients who could have been surgically treated. Another question is about the objectivity of PS evaluation. In ECOG, PS 1 is defined as 'Restricted in physically strenuous activity but ambulatory and able to carry out work of a light or sedentary nature'. When considering the average age of HCC patients, determining PS as PS 0 and 1 is not clinically easy. Therefore, in actual clinical practice, the treatment strategy is chosen based on tumor progression and liver function rather than PS.

There might be a drawback in the BCLC guidelines. Regardless of tumor status or liver function, patients with PS1 are classified as BCLC stage 3 and are recommended to be treated with systemic chemotherapy $(11,12)$. This classification does not suggest an opportunity for more diverse treatments for HCC patients with PS 1. Indeed, several studies have consistently showed that patients with PS1 have superior outcomes following treatments other than sorafenib. Therefore, it is necessary to revise the part corresponding to PS in the BCLC guidelines. Another limitations of the BCLC staging system is that treatment is suggested according to the CTP class, and even patients with the same CTP class B may have different prognosis depending on the difference in CTP scores. This is the basis for arguing that subclassification is necessary in HCC patients with BCLC stage B and C. It seems to be a good proposal to modify and subdivide the BCLC stage by comprehensively evaluating the PS, CTP, tumor extent, and presence of extrahepatic spread as described by Cho et al. (13), but further research is warranted before this can be implemented.

In conclusion, because the treatment of advanced liver cancer has not yet been established, extensive studies on various treatment methods are needed. This article shows data indicating a better prognosis in the patients treated with TACE, against the recommendations of existing guidelines for the treatment of BCLC stage C patients with PS1 but no vascular invasion or extrahepatic spread. Likewise, even for HCC patient with PS1, more aggressive locoregional treatments are expected to improve prognosis. In addition, it is worth considering a careful revision of BCLC staging and treatment recommendations for HCC patients with a relatively good PS.

\section{Acknowledgments}

Funding: None.

\section{Footnote}

Conflicts of Interest: Both authors have completed the ICMJE uniform disclosure form (available at http://dx.doi. org/10.21037/atm-20-4316). The authors have no conflicts of interests to declare.

Ethical Statement: The authors are accountable for all aspects of the work in ensuring that questions related to the accuracy or integrity of any part of the work are appropriately investigated and resolved.

Open Access Statement: This is an Open Access article distributed in accordance with the Creative Commons Attribution-NonCommercial-NoDerivs 4.0 International License (CC BY-NC-ND 4.0), which permits the noncommercial replication and distribution of the article with the strict proviso that no changes or edits are made and the original work is properly cited (including links to both the formal publication through the relevant DOI and the license). See: https://creativecommons.org/licenses/by-nc-nd/4.0/.

\section{References}

1. Forner A, Llovet JM, Bruix J. Hepatocellular carcinoma. Lancet 2012;379:1245-55.

2. Finn RS, Zhu AX, Farah W, et al. Therapies for advanced stage hepatocellular carcinoma with macrovascular 
invasion or metastatic disease: A systematic review and meta-analysis. Hepatology 2018;67:422-35.

3. Nishikawa H, Kita R, Kimura T, et al. Clinical implication of performance status in patients with hepatocellular carcinoma complicating with cirrhosis. J Cancer 2015;6:394-402.

4. Hsu CY, Lee YH, Hsia CY, et al. Performance status in patients with hepatocellular carcinoma: determinants, prognostic impact, and ability to improve the Barcelona Clinic Liver Cancer system. Hepatology 2013;57:112-9.

5. Giannini EG, Bucci L, Garuti F, et al. Patients with advanced hepatocellular carcinoma need a personalized management: A lesson from clinical practice. Hepatology 2018;67:1784-96.

6. Aziz AO, Omran D, Nabeel MM, et al. Aggressive Treatment of Performance Status 1 and 2 HCC Patients Significantly Improves Survival - an Egyptian Retrospective Cohort Study of 524 Cases. Asian Pac J Cancer Prev 2016;17:2539-43.

7. Hsu CY, Liu PH, Lee YH, et al. Aggressive therapeutic strategies improve the survival of hepatocellular carcinoma patients with performance status 1 or 2: a propensity score analysis. Ann Surg Oncol 2015;22:1324-31.

Cite this article as: Yu JH, Lee JW. Limitations of the Barcelona clinic liver cancer staging treatment strategy in hepatocellular carcinoma patients with performance status 1 . Ann Transl Med 2020;8(17):1042. doi: 10.21037/atm-20-4316
8. Zhao S, Dou W, Fan Q, et al. Identifying optimal candidates of transarterial chemoembolization (TACE) vs. sorafenib in patients with unresectable hepatocellular carcinoma. Ann Transl Med 2020;8:587.

9. da Motta Girardi D, Correa TS, Crosara Teixeira M, et al. Hepatocellular Carcinoma: Review of Targeted and Immune Therapies. J Gastrointest Cancer 2018;49:227-36.

10. El Dika I, Khalil DN, Abou-Alfa GK. Immune checkpoint inhibitors for hepatocellular carcinoma. Cancer 2019;125:3312-9.

11. Omata M, Cheng AL, Kokudo N, et al. Asia-Pacific clinical practice guidelines on the management of hepatocellular carcinoma: a 2017 update. Hepatol Int 2017;11:317-70.

12. European Association for the Study of the Liver. EASL Clinical Practice Guidelines: Management of hepatocellular carcinoma. J Hepatol 2018;69:182-236.

13. Cho HJ, Kim SS, Kang SY, et al. A Proposal for Modification of the Barcelona Clinic Liver Cancer Staging System Considering the Prognostic Implication of Performance Status. Gut Liver 2019;13:557-68. 